\title{
EVALUACIÓN DEL EFECTO DE TRES NIVELES DE TOSTIÓN EN LA CALIDAD EN TAZA DE ALGUNAS VARIEDADES DE CAFÉ DE LA ESPECIA COFFEA ARABICA L
}

\author{
EVALUATION OF THE EFFECT OF THREE LEVELS OF ROASTING QUALITY ON \\ THE CUP IN SOME COFFEE VARIETIES OF THE SPECIE COFFEA ARABICA L
}

Rosa María Méndez Parra¹, Paulo César Carmona Tabares², Oscar Emilio Molina Díaz.

${ }^{1}$ Licenciatura en Matemáticas. Seminario Interdisciplinario Grupo en Matemática Aplicada (SIGMA). Facultad de Educación. Universidad del Quindío. Armenia, Quindío. Colombia.

${ }^{2}$ Maestría en Biomatemáticas. Seminario Interdisciplinario Grupo en Matemática Aplicada (SIGMA).

Facultad de Ciencias Básicas y Tecnologías. Universidad del Quindío. Armenia, Quindío. Colombia.

Corresponding Author: Rosa María Méndez Parra, celular 3007848595,

correo: rosamendez@uniquindio.edu.co

\section{RESUMEN}

El café es una de las bebidas más consumidas en el mundo, y Colombia tiene cualidades excepcionales para su cultivo, producción e industrialización. El objetivo de este trabajo fue evaluar mediante catación, la calidad en taza de cuatro variedades de café, de origen colombiano (exactamente del Departamento del Quindío), de la especie Coffea arabica L. Se realizó un estudio de tipo cualitativo- y descriptivo, apoyado en un diseño experimental de dos factores, para el cual se escogieron las variedades: Geisha, Bourbon rosado, Moka y Castillo, los tres primeros son cafés especiales y el último es una variedad de porte medio, cuya calidad en taza es comparable con las otras. Para la evaluación en taza, se realizaron tres niveles de tostión; se encontró que la variedad Geisha obtuvo la mayor puntuación en sus tres niveles de tostión, y entre éstos, el nivel de tostión bajo resaltó más sus cualidades. Finalmente, se concluye que la variedad del café es uno de los factores que influye en la calidad de taza.

Palabras clave: cafés diferenciados, catación, niveles de tostión.

Cómo citar:

Méndez Parra, R., Carmona Tabares, P \& Molina Díaz, O. (2019) CALIDAD EN TAZA DE ALGUNAS VARIEDADES DE CAFÉ DE LA ESPECIE COFFEA ARABICA L. Revista de Investigaciones Universidad del Quindío. Vol 31, pp.54-61.

Información del artículo: Recibido: 28 mayo 2019; Aceptado: 5 agosto 2019 


\begin{abstract}
Coffee is one of the most consumed beverages in the world, and Colombia has exceptional qualities for its cultivation, production and industry. The objective was to evaluate using a tasting panel, the quality in a cup of four varieties of coffee, of colombian origin (exactly from the Department of Quindío), of the species Coffea arabica L. A quantitative - descriptive study was carried out, supported in an experimental design, the selected varieties: Geisha, pink Bourbon, Moka and Castillo were chosen, the first three are special coffees and the last is a seed, of superior quality, comparable to the others. For the cup evaluation, different levels of roasting were performed. It was found that for the Geisha variety obtained the highest score in its three levels of roasting, and among these, the level of low roasting highlighted its qualities. It's concluded that for the varieties used, the variety of coffee influences the cup quality.
\end{abstract}

Keywords: Tasting panel, differentiated coffees, roasting levels.

\section{INTRODUCCIÓN}

El trabajo realizado consistió en evaluar la calidad en taza de cuatro (4) variedades de café, de la especia Coffea arabica L. dos de porte alto (Geisha, y Bourbon Rosado) y dos de porte bajo (Moka y Castillo), los tres primeros son cafés especiales o diferenciados y el último es una variedad que, por su porte superior, calidad y manejo en el beneficio, es comparable con las otras.

En este trabajo se presentan los resultados de un diseño experimental (Montgomery, 2017), que se realizó bajo condiciones homogéneas (altitud, humedad, $\mathrm{pH}$, merma durante el proceso de tostión, entre otros); las cuatro variedades que se caracterizan por su calidad, se evaluaron, de acuerdo a tres niveles de tostión, esto es, se determinó cuál nivel de tostión realza las características en las cuatro variedades de café

El proceso de tostado es clave en la cadena productiva del café, toda vez que durante éste se desarrollan diversas reacciones químicas y físicas que alteran, recrean, modifican o realzan la calidad en taza (Puerta, 2011).

El café pertenece al género coffea, y a la familia de las rubiáceas (Rubiaceae). Este género tiene dos importantes especies: arábica y canephora (o robusta), las cuales poseen sus propias cualidades, cultivándose en climas y altitudes distintas, consumiéndose más, a nivel mundial, la especie Coffea arábica (Puerta, 2000).

La calidad en taza de la bebida del café está liga- da a los procesos que hay antes de su preparación: Clima, suelo, manejo del cultivo, recolección, beneficio, trilla, tueste, molienda y preparación, son todos eslabones en la cadena productiva del café y cuyas particularidades merecen ser estudiadas y llevadas con altos niveles de calidad para garantizar una taza de propiedades organolépticas excelentes.

El cultivo comercial del café en Colombia es una actividad agrícola e industrial que ha tenido una importancia social y económica significativa. Actualmente nuestro país exige para la comercialización de café pergamino seco un contenido final de humedad entre el 10-12\%, con el fin de impedir la proliferación de microorganismos y la actividad enzimática que pueden deteriorar el grano durante su almacenamiento.

Dentro de las etapas de la cadena productiva de la industria cafetera, la del tostado viene siendo estudiada desde hace ya varios años, para determinar cómo es la dinámica de la transferencia de calor desde el cilindro o tambor donde se tuesta, al interior del grano, toda vez que en la tostación el sabor natural del grano puede ser recreado o modificado con el fin de buscar una excelente calidad en taza.

El café colombiano cuenta con ventajas competitivas en el mercado internacional y, de hecho, se les reconoce un sobreprecio debido a varios factores como su origen geográfico, las respectivas condiciones climáticas y de suelos de sus cultivos, su origen botánico, especie y variedades sembradas, la cultura cafetera del país y los pro- 
cesos realizados hasta la obtención del producto para exportación y consumo (Puerta, 2013).

En síntesis, hablar de la calidad del café consiste en establecer un equilibrio en la diversidad de sus atributos y características. Un café de calidad se caracteriza por ser una bebida con una taza limpia, con acidez y cuerpo medio-alto, aroma pronunciado y completo.

En el mercado mundial se manejan dos grupos principales de cafés: la calidad común y la calidad de los cafés especiales, aproximadamente el $80 \%$ del café comercializado corresponde a cafés de calidad común (Van Hilten, 2011), (Ponte, 2002). Para la Asociación de Cafés Especiales, S.C.A. (por sus siglas en inglés), al igual que para la Federación Nacional de Cafeteros, los cafés especiales son aquellos que conservan una consistencia en sus características físicas, sensoriales, prácticas culturales en su beneficio y en sus procesos finales; todas ellas corresponden a cualidades que los diferencian de los cafés comunes y por las cuales los clientes están dispuestos a pagar un precio superior (Arcila et al, 2007).

En el proceso de tostado se somete el grano de café al calor, el grano varía su coloración de verde a amarillo, luego a marrón y finalmente a un color más oscuro. Durante este proceso, que puede durar hasta 15 minutos con máquinas tostadoras trabajando entre $100^{\circ} \mathrm{C} \mathrm{y} 300^{\circ} \mathrm{C}$ aproximadamente, se presentan dos momentos de "explosiones", los cuales se utilizan como indicadores del nivel de tostión alcanzado. En el momento que la temperatura interior del grano alcanza aproximadamente $\operatorname{los} 180^{\circ} \mathrm{C}$ se liberan los aceites que realzan el sabor del café y además se liberan grasas, azúcares y proteínas (cambios químicos). También, a medida que se tuesta el café, el grano va ganando volumen y perdiendo humedad (cambios físicos). Como conclusión tenemos que la variación de la temperatura y el tiempo de tostado del grano generan cambios en el color y el sabor del mismo (Giner, 2008).

La temperatura es fundamental durante la tostión del café en virtud a que ella es responsable de las modificaciones que se puedan producir en las propiedades propias del grano, por esta razón es posible perfilar mediante el proceso de tostado la calidad de la taza. En ese sentido varios autores manifiestan la importancia de este eslabón en la cadena productiva de la industria cafetera.

\section{METODOLOGÍA}

Se seleccionaron granos, de la parte productiva de la planta (normalmente parte media), maduros y sobre maduros nada más y del mayor tamaño en cada caso. Las muestras de café fueron beneficiadas de la siguiente manera: el despulpado fue manual, la fermentación con la cantidad mínima de agua utilizando el método Fermaestro (Peñuela A. et al, 2014), secado al sol y trillado a mano, características propias de café tipo semilla para siembra. Al café Moka no le fue removido su mucílago para el secado y de esta manera, producir un café tipo honey (acaramelado). No obstante, esta variedad no fue tenida en cuenta al momento de la evaluación de los resultados ya que fue descalificada, resultando así un proceso de valoración de resultados para los cafés restantes.

Se contaron con muestras de 500 gramos de las variedades Geisha, Bourbon rosado, Moka y Castillo, procedentes de fincas de los municipios de Circasia y Pijao, en el Departamento del Quindío, con altitudes que varían de 1500 m.s.n.m. a 1800 m.s.n.m. y temperaturas promedios que varían entre $18^{\circ} \mathrm{C}$ a $22^{\circ} \mathrm{C}$; se realizó un diseño experimental de dos factores, factor 1: variedad (Geisha, Bourbon rosado, Moka y Castillo) y factor 2: proceso de tostión (bajo, medio y alto); de los $500 \mathrm{~g}$ se seleccionaron tres muestras de $100 \mathrm{~g}$ cada una, las cuales fue sometida a un procesos de tostión diferente: bajo, medio o alto, iniciando los procesos a una temperatura de $150^{\circ} \mathrm{C}, 170^{\circ} \mathrm{C}$ y $190^{\circ} \mathrm{C}$, respectivamente. En todos los casos, se concluyó el proceso buscando un nivel medio de tostación para catación de cafés especiales según escala (AGTRON de 45-55). Para la tostión se usó una tostadora marca Quantik TC 300 ARProfile, (dos tambores) y la medición del pH de 
las variedades se hizo en el Laboratorio del Grupo de Investigación de Frutos Tropicales, de la Universidad del Quindío.

Se hizo un análisis sensorial de cada una de las muestras de 100 g. (3 repeticiones por cada proceso de tostado, y tres procesos de tostado por cada variedad, en total 36 tazas fueron valoradas) con el apoyo de un profesional del Servicio Nacional de Aprendizaje "SENA", (por falta de recurso profesional al momento de la catación no se pudo contar con más catadores), se realizó de forma que la calificación se dio sin conocer la variedad y nivel de tostión de donde provenían las tazas; se utilizó agua de excelente calidad en la proporción de $150 \mathrm{~mL}$ para $8 \mathrm{~g}$ de café tostado y molido, usando para la evaluación la hoja de catación propuesta por la S.C.A (el Contrato Q del Coffee Quality Institute, utiliza éste formato para todas sus evaluaciones), la catadora evaluó las propiedades organolépticas del café y calificaron de forma cualitativa y cuantitativa (en una escala de 6 a 10) los siguientes aspectos: fragan- cia/aroma, acidez, cuerpo, sabor, sabor residual, balance, puntaje total. Por otros métodos se evaluaron peso, densidad, humedad, ancho, largo y peso de los granos, merma después del proceso de tostión y $\mathrm{pH}$ de la bebida, para todas estas variables se hizo estadística descriptiva. La variable Puntaje total fue valorada con análisis estadístico mediante la prueba no paramétrica de KruskalWallis.

\section{RESULTADOS}

Los valores promedios de humedad, densidad y pH, son dadas en la Tabla 1, las medidas promedio de largo, ancho y peso son dadas en la Tabla 2 y la pérdida de peso en porcentaje, para cada una de las variedades tostadas es detallada en la Tabla 3; de la información contenida en ellas, se deriva que la variedad con mayor densidad de grano es el Bourbon rosado y los valores de humedad de todas las variedades, son altos comparados con el valor ideal del $12 \%$.

Tabla 1 Medidas de humedad, densidad y pH según variedad.

\begin{tabular}{|c|c|c|c|}
\hline Variedad & Humedad \% & Densidad $\mathbf{( g / m l )}$ & $\mathbf{p H}$ \\
\hline Geisha & 15,2 & 677 & 5,11 \\
\hline Bourbon rosado & 15,7 & 674 & 5,05 \\
\hline Castillo & 14,9 & 714 & 5,06 \\
\hline Moka & 9,4 & 657 & 5,12 \\
\hline
\end{tabular}

Tabla 2: Medidas de los granos de café acorde a la variedad.

\begin{tabular}{|c|c|c|c|}
\hline Variedad/medidas & Largo $\mathbf{( m m})$ & Ancho $\mathbf{( m m})$ & Peso $(\mathbf{g})$ \\
\hline Geisha & 10,2 & 7,3 & 0,2 \\
\hline Bourbon rosado & 10,6 & 6,8 & 0,2 \\
\hline Castillo & 10,3 & 7,0 & 0,2 \\
\hline Moka & 7,0 & 6,6 & 0,12 \\
\hline
\end{tabular}

Tabla 3: Porcentaje de pérdida de peso según variedad y nivel de tostión.

\begin{tabular}{|c|c|c|c|}
\hline Variedad/ niveles de tostión & Bajo (\%) & Medio (\%) & Alto (\%) \\
\hline Geisha & 32,9 & 18,0 & 18,1 \\
\hline Bourbon rosado & 19,2 & 33,2 & 19,8 \\
\hline Castillo & 31,5 & 17,6 & 16,6 \\
\hline Moka & 28,7 & 12,6 & 11,8 \\
\hline
\end{tabular}


La Tabla 4 resume la información del análisis estadístico no paramétrico para la variable Puntaje total, el cual fue realizado mediante prueba de Kruskal-Wallis (Peña, 2010), y en el que se encontró que, para las muestras usadas, existe una diferencia significativa entre las medianas de la variable Puntaje total, de las variedades Geisha,

Tabla 4. Prueba de Kruskal-Wallis para Puntuaje total

\begin{tabular}{|r|c|c|}
\hline Variedad & Tamaño muestra & Rango promedio \\
\hline Geisha & 3 & 7,83333 \\
\hline Bourbon & 3 & 3,0 \\
\hline Castillo & 3 & 4,16667 \\
\hline
\end{tabular}

Estadístico $=5,13165$ Valor-P $=0,0768556$ Bourbon rosado y Castillo, asumiendo un $\alpha=0,1$.

La Figura 1 corresponde al gráfico de cajas, en el cual se observa que el 50\% del café tostado de la variedad Geisha, tiene puntaje total promedio, mayor que las demás variedades, para los tres niveles de tostión.

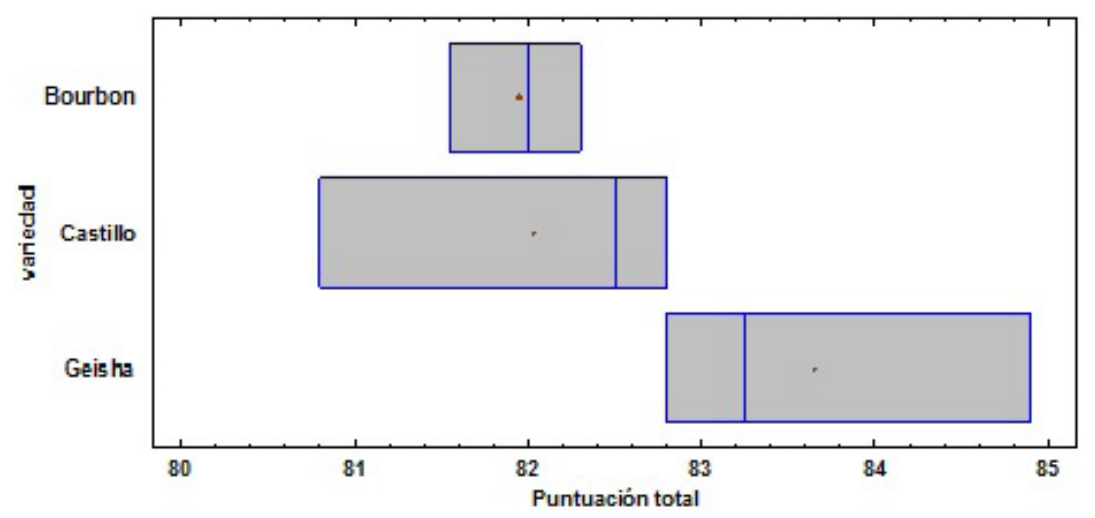

Figura 1: Gráfico de cajas para la variable Variedad vs Puntaje total.

La Figura 2 corresponde al gráfico de medias, en el cual se evidencia que la variedad Geisha tiene una calificación más alta en el promedio de los puntajes totales de los tres procesos de tostión, frente al Bourbon rosado y Castillo.

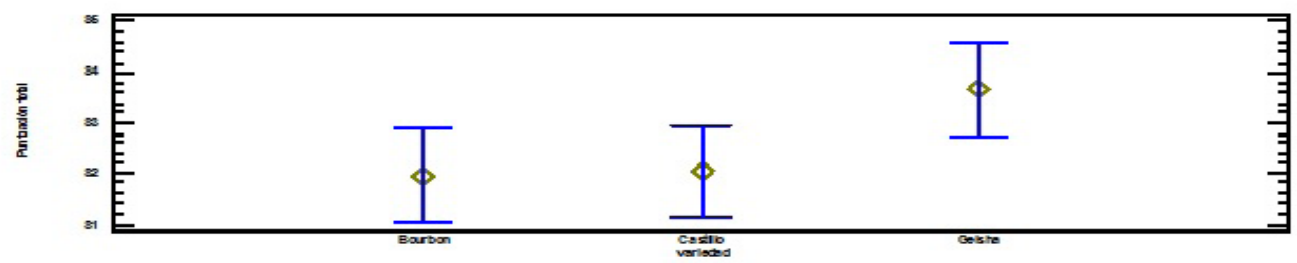

Figura 2: Gráfico de medias para la variable Puntaje total vs Variedad.

Todas las muestras presentan características de suavidad, como es de esperarse en un café colombiano, algunas sobresalen por su fragancia/ aroma (Geisha y Bourbon rosado, ambos en nivel medio de tostión), otras por su dulzor (Castillo en su nivel de tostión alto).

Los resultados de la evaluación de catación en general, destacan la variedad Geisha y entre todos los niveles, el nivel de tostión bajo resaltó más las cualidades del mismo, lo cual reafirma lo que usualmente se sugiere para esta variedad en cuanto al nivel de tueste. La variedad Moka fue descalificada ya que presentó un olor a fenol en el aroma, el cual se confirmó en la bebida (posiblemente por un exceso de tiempo de fermentación) 
y las restantes variedades obtuvieron calificaciones y puestos como se detalla a continuación en la Tabla 5.

Tabla 5: Calificación de las variedades según la catación.

\begin{tabular}{|l|l|l|l|l|l|l|}
\hline $\begin{array}{l}\text { Nivel de tostión/ } \\
\text { puntaje de calidad } \\
\text { según variedad }\end{array}$ & Geisha & \multicolumn{2}{l|}{ Bourbon rosado } & \multicolumn{2}{l|}{ Castillo } \\
\cline { 2 - 8 } & Calificación & Puesto & Calificación & Puesto & Calificación & Puesto \\
\hline Bajo & 85 & 1 & 81,5 & 7 & 80,8 & 8 \\
\hline Medio & 83,25 & 2 & 82,3 & 5 & 82,8 & 3 \\
\hline Alto & 82,8 & 3 & 82 & 6 & 82,5 & 4 \\
\hline
\end{tabular}

Detallando el tiempo del proceso de tostión se tiene que lo recomendable es que el grado de humedad del grano sea del 12\%; ahora, en relación a los tres niveles de tostión para cada una de las cuatro variedades, se encuentra que las muestras de Moka y Bourbon rosado, el tiempo de tostión en el nivel bajo es más prolongado que los niveles medio y alto (10:00 y 13:30 minutos, respectivamente), en tanto que para las variedades Geisha y Castillo el nivel que mayor tiempo emplea para tostar el café es el medio (10:30 y 11:00 minutos, respectivamente). En este sentido (Giner, 2012) afirma que el tiempo del tostado depende de varios factores, entre los más importantes están: hábito de consumo, tipo de tostadora, variedad de café y usos del producto resultante.

En lo relacionado con el proceso de tostión en sus tres niveles y la pérdida de peso en porcentaje, se puede afirmar de las muestras tostadas lo siguiente: entre más aumenta el nivel de tostión, es menor el porcentaje de pérdida de peso (excepto para la variedad Bourbon rosado en su nivel medio, que pierde el $33,2 \%$ y en su nivel bajo pierde un $19 \%$ de su peso), esto es, hay mayor pérdida de peso en el nivel de tostión bajo para las cuatro variedades y de ellas, el café Geisha tiene el mayor porcentaje $(32,9 \%)$, en tanto que para el nivel de tostión alto, en las cuatro variedades, se presenta el menor porcentaje de pérdida de peso. El café Moka presenta el porcentaje más pequeño (11,8\%). Ahora, descartando la variedad Moka por el defecto que presentó, resulta que el café Castillo es la variedad de menor porcentaje de pérdida en el nivel tostión alto (16,6\%).

Las notas encontradas en fragancia / aroma y sabores percibidos según las variedades son detallados en la Tabla 6.

Tabla 6: Aromas y sabores de las variedades según catación

\begin{tabular}{|l|l|l|}
\hline Variedad y nivel de tostión & Fragancia -Aroma & Sabor \\
\hline Geisha - Bajo & limoncillo, vainilla & limoncillo, panela \\
\hline Geisha - Mediano & floral, coctel de frutas, vainilla & limoncillo, caramelo \\
\hline Geisha - Alto & floral, cítrico, frutoso & dulce \\
\hline Castillo - Bajo & cereal, madera & \\
\hline Castillo - Mediano & nuez, madera & dulce, madera \\
\hline Castillo - Alto & nuez, almendra & panela, cereal \\
\hline Bourbon - Bajo & chocolate, maní, nuez & chocolate \\
\hline Bourbon -mediano & cítrica, vainilla & nuez, chocolate, afrutado \\
\hline Bourbon - Alto & floral, cítrica, amareto & caramelo, almendra \\
\hline
\end{tabular}


Finalmente, se presenta una caracterización descriptiva de las propiedades organolépticas de cada una de las muestras que en cada una de las variedades obtuvieron la mayor puntuación. En las Figuras 3-5, se resume el resultado del proceso de catación, de las cuales se puede decir que la variedad Geisha en el nivel bajo de tostión resultó con una mayor acidez y mayor cuerpo que las otras dos variedades, en tanto que la valoración de la variable fragancia-aroma, la variedad Castillo fue menos calificada que las otras dos. En general, la variedad Geisha obtiene mayor calificación en cada una de las variables.

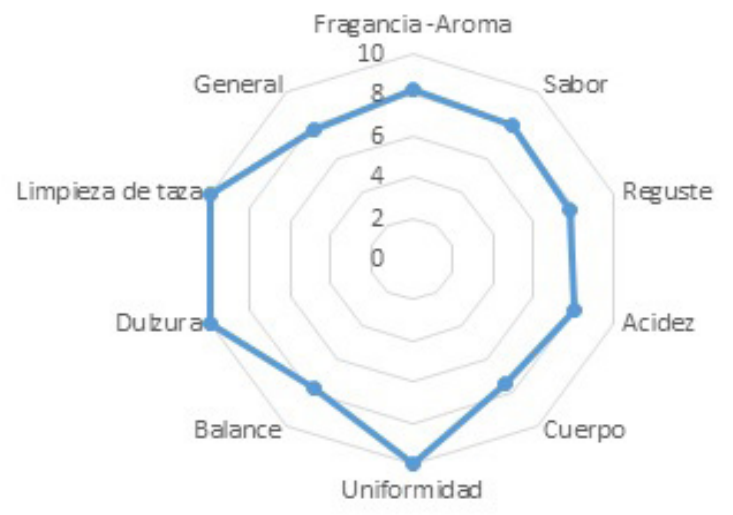

Figura 3. Caracterización de la variedad Geisha nivel bajo.

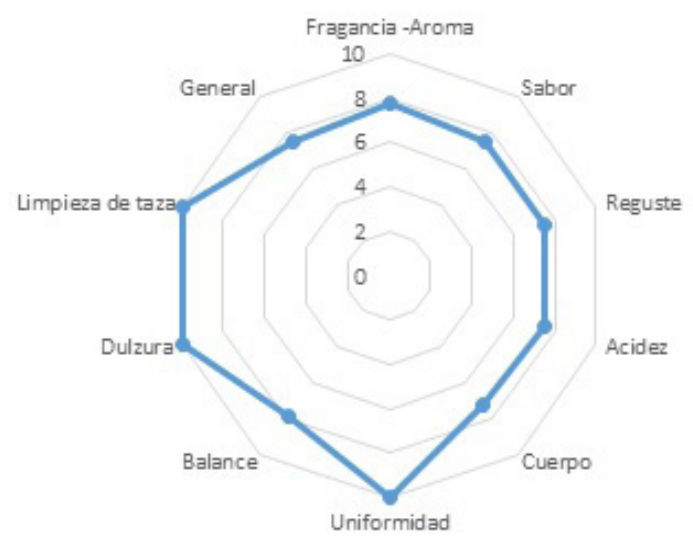

Figura 4. Caracterización de la variedad Castillo nivel alto.

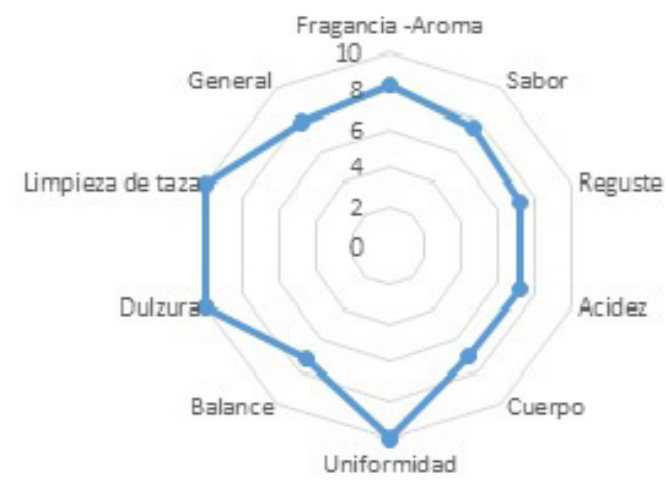

Figura 5. Caracterización de la variedad Bourbon nivel medio.

\section{DISCUSIÓN}

Con este trabajo se corrobora la suavidad y dulzura de la especie Coffea arabica L. Se confirma además, que las indicaciones que se tienen para el nivel de tostión apropiado de los cafés según las variedades utilizadas, el nivel de tostión bajo para el Geisha es el adecuado para destacar la calidad de las propiedades organolépticas del mismo y que en la variedad Castillo y Bourbon rosado, el nivel de tostión medio, es el más adecuado o aconsejado para resaltar las propiedades que dan lugar a una taza de calidad, lo cual se puede verificar en las mayores puntuaciones obtenidas (Tabla 5).

También se resaltan los sabores afrutados de las variedades Geisha y Bourbón rosado.

Con la prueba estadística de Kruskal-Wallis y el resultado del proceso de catación, se puede concluir para las tres muestras evaluadas, que la variedad del café, realmente influye en el puntaje total de la taza.

Este tipo de estudios es relevante por cuanto permite a los caficultores y demás personas que obtienen su sustento de este pilar de la economía regional y del país, conocer aspectos que mejoran la calidad del café que producen y/o procesan. 


\section{AGRADECIMIENTOS}

Los autores agradecen a la Vicerrectoría de Investigaciones de Universidad del Quindío, a la Licenciatura en Matemáticas, al programa de
Física y la Maestría en Biomatemáticas, por el apoyo en el desarrollo y la divulgación de los resultados (Proyecto de investigación No. 781) y a María Paula Pinzón y Diana Milena Galvis, por las asesorías recibidas.

\section{REFERENCIAS}

1. Giner, J. (2012). El Tueste de Café. Fórum Café, 6-15.

2. Montgomery, D. (2017). Design and Aanalysis of Experiments. Jhon Wiley \& Sons.

3. P.J. Arcila, F. Moreno, L.F. Salazar, E. Hincapié. (2007). Sistemas de producción de café en Colombia. Centro de Investigaciones CENICAFE.

4. Peña, D. (2002). Regresión y diseño de experimentos. Alianza.

5. Peña, D. (2010). Regresión y diseño de experimentos. Alianza.

6. Peñuela A. et al. (2014). Método fermaestro: Para determinar la finalización de la fermentación del mucílago de café. Centro Nacional de Investigaciones de Cafe CENICAFE.

7. Ponte, S. (2002). Estándares, comercio y equidad: Lecciones de la industria de los cafés especiales. Economía Mundial del Café. Centro de investigaciones para el desarrollo de Copenhague, 131-163.

8. Puerta, G. I. (2000). Calidad en taza de algunas mezclas de variedades de café de la especie Coffea arabica L. Cenicafé, 5-19.

9. Puerta, G. I. (2011). Composición química de una taza de café. Avances Técnicos Cenicafé, 1-12.

10. Puerta, G. I. (2013). Especificaciones de origen y buena calidad del café de Colombia. Centro de Investigaciones de Café CENICAFE.

11. Puerta, G. I. (2013). Especificaciones de origen y buena calidad del cafe de Colombia. . Centro de investigación del café CENICAFE.

12. Schwartzberg, H. (2002). Modeling Bean Heating During Batch Roasting of Coffee Beans. Boca Raton, EEUU: CRC Press.

13. Van Hilten, H. J., Fisher, P. J., \& Wheeler, M. A. (2011). The Coffee Exporter's Guide. Geneva, Switzerland: International Trade Centre UNCTAD/GATT.

14. W.N. Herna' ndez-Dí az, I.I. Ruiz-Lo' pez, M.A. Salgado-Cervantes, G.C. Rodrí guez-Jimenes. (2008). Modeling heat and mass transfer during drying of green coffee beans using prolate spheroidal geometry. Journal of Food Engineering, 1-9. 